\title{
Ecuaciones de niveles de humedad relacionada a la densidad básica de la madera de especies forestales tropicales en Madre de Dios, Perú
}

\author{
Equations of humidity levels related to the basic wood density of tropical forest species \\ in Madre de Dios, Peru
}

Emer-Ronald Rosales-Solórzano

\begin{abstract}
Scarce information in physical studies of the wood made tests on 10 representative forest species to determine mathematical equations, in addition 95 other species were selected where models were applied, tested and validated for moisture content equilibrium (CHE), free moisture content (CHL), fiber saturation point (PSF) and maximum moisture content $(\mathrm{CHM})$ of the wood, using the instant measurement method prior saturation in hot water at $100^{\circ} \mathrm{C}$ for 24 hours and drying in an oven. Basic density (DB) varied from 0.27 to $0.92 \mathrm{~g} / \mathrm{cm}^{3}$, where the measured range $\mathrm{CHM}$ ranged from $49-302 \%$ is located, while the set had an exponential behavior equal to $\mathrm{CHM}$ $=597.96 \mathrm{e}^{-2.843 \mathrm{DB}}$ that ranged from 44 to $281 \%$. The estimated $\mathrm{CHL}$ changed from 31 to $234 \%$ and showed an exponential behavior set equal to $\mathrm{CHL}=583.84 \mathrm{e}^{-3.263 \mathrm{DB}}$ changed from 29 to $245 \%$. The PSF estimated average was $27 \%$ ranging from 18 to $68 \%$ and adjusted behavior exhibited a potential equal to PSF $=14.678 \mathrm{DB}^{-0.83}$ that ranged from 16 to $44 \%$. The average measured CHE was $12.63 \%$ which ranged from 9 to $16 \%$ and a logarithmic behavior tube set equal to $\mathrm{CHE}=-4.141 \mathrm{ln}(\mathrm{DB})+9.8939$ which varied from 10 to $15 \%$. The generated models predicted humidity levels with a very high correlation coefficient $(r)$ in $\mathrm{CHM}$ and $\mathrm{CHL}$ equal to $r=0.991$, high in the PSF of $r=0.828$ and CHE with $r=0.851$.
\end{abstract}

Key words: Humidity level, basic density, wood, Madre de Dios, Perú.

1. Escuela Profesional de Ingeniería Forestal y Medio Ambiente. Facultad de Ingeniería. Universidad Nacional Amazónica de Madre de Dios, Madre de Dios. Perú, errs1973@gmail.com 


\section{Resumen}

La escasa información en estudios físicos de la madera hicieron realizar ensayos en 10 especies forestales representativas para determinar ecuaciones matemáticas, además se seleccionaron otras 95 especies donde se aplicaron, probaron y validaron los modelos para el contenido de humedad equilibrio (CHE), contenido de humedad libre (CHL), punto de saturación de las fibras (PSF) y contenido de humedad máxima (CHM) de la madera, usando el método de medición instantánea previa saturación en agua caliente a $100{ }^{\circ} \mathrm{C}$ por 24 horas y secado en estufa. La densidad básica (DB) varió de 0,27 a $0,92 \mathrm{~g} / \mathrm{cm}^{3}$, rango donde se ubica el CHM medido que cambió de 49 a $302 \%$, mientras que el ajustado tuvo un comportamiento exponencial igual a $\mathrm{CHM}=597,96 \mathrm{e}^{-2,843 \mathrm{DB}}$ que varió de 44 a $281 \%$. El CHL estimado cambió de 31 a $234 \%$ y el ajustado presentó un comportamiento exponencial igual a $\mathrm{CHL}$ $=583,84 \mathrm{e}^{-3,263 \mathrm{DB}}$ que cambió de 29 a $245 \%$. El PSF estimado promedio fue $27 \%$ variando de 18 a $68 \%$ y el ajustado exhibió un comportamiento potencial igual a PSF $=14,678 \mathrm{DB}^{-0,83}$ que varió de 16 a $44 \%$. El CHE medido promedio fue $12,63 \%$ variando de 9 a $16 \%$ y el ajustado tuvo un comportamiento logarítmico igual a $\mathrm{CHE}=-4,141 \mathrm{ln}(\mathrm{DB})+9,8939$ que cambió de 10 a 15 $\%$. Los modelos generados predijeron los niveles de humedad con un coeficiente de correlación ( $r$ ) muy alta en $\mathrm{CHM}$ y $\mathrm{CHL}$ igual a $r=0,991$, alta en el PSF de $r=$ 0,828 y CHE con $r=0,851$.

Key words: Nivel de humedad, densidad básica, madera, Madre de Dios, Perú.

\section{Introducción}

El contenido de humedad en árboles vivos puede variar entre 30 y más de $400 \%$, tal variación está en función de la especie, condiciones de crecimiento y densidad básica de su madera. Conocer el contenido de humedad de la madera a secar no necesariamente indica la cantidad de agua a extraer, ya que un mismo nivel de contenido de humedad podrá significar cantidades de agua muy diferentes, dependiendo de la densidad básica que caracterice a una determinada madera [7], [12], [21], [23]. La bibliografía demuestra que generalmente para una especie de mayor o menor densidad básica se tendrá un nivel de humedad menor o mayor, respectivamente [1], [7]. Esto indica que la humedad de la madera depende, no sólo de las condiciones ambientales, sino también, de las características intrínsecas de las especies maderables. Así como también, la diferencia entre albura y duramen en una misma especie y la cantidad de extractivos [5], [16], [20], [23].

El contenido de humedad libre (CHL) o agua libre, llena el lumen o cavidad de las células o tubos (vasos, fibras, etc.), es absorbido por capilaridad. El agua libre una vez perdida por la madera, ya no puede ser recuperada a partir de la humedad atmosférica para recuperarla, habrá de ser por inmersión directa en el agua. El porcentaje de agua libre en un estudio varía de 14 a $238 \%$ para un rango de densidad básica de 0,29 a 1,05 g/ $/ \mathrm{cm}^{3}$, valores que en los procesos de impregnación representaría el máximo de solución preservante que es capaz de absorber una madera y que en los procesos de secado representa el agua que se puede eliminar por capilaridad sin el requerimiento de elevada energía [8], [23].

El contenido de humedad máxima (CHM) o cantidad de agua máxima, están completamente saturados de agua la pared celular como los lúmenes o cavidades [23]. Investigaciones hechas por [15], [21] reportan un rango del CHM de $44 \%$ para maderas pesadas nativas de Estados Unidos con densidad de $0,90 \mathrm{~g} / \mathrm{cm}^{3}$ a $267 \%$ para maderas ligeras con densidad de $0,30 \mathrm{~g} / \mathrm{cm}^{3}$, sin embargo, indican que en urban (Krugiodendron ferrum Vahl) con una densidad de 1,18 alcanza un contenido de humedad máxima de $26 \%$ y en contraste topa (Ochroma lagopus Sw) tiene una densidad de 0,2 pudiendo llegar a presentar $400 \%$ o más [23].

El punto de saturación de las fibras (PSF), el mínimo asciende a $22 \%$ y el máximo a unos $35 \%$ o valores de hasta $50 \%$ en maderas de raíz muy ligeras y de capilares extraordinariamente pequeños [7]. Estudios en Canadá, se descubre que la evacuación del agua higroscópica comienza a partir de un contenido de humedad de 51 $\%$ en Cedrelinga catenaeformis (Ducke) Ducke (tornillo), $36 \%$ en Aspidosperma macrocarpon Mart. (pumaquiro), $77 \%$ en Ormosia sp. (huayruro) y $14 \%$ en Acer rubrum L. (arce), probando que la contracción comienza a partir de estos contenidos de humedad y no a partir de $30 \%$, esto significa que los cambios físico mecánicos de la madera pueden darse a elevados y bajos contenidos de humedad [1], [7], [17]. Hay que considerar que la causa principal de las apreciables diferencias halladas en los distintos PSF es la formación de duramen de algunas maderas producida por el almacenamiento de resinas, taninos, y materias colorantes en los espacios inter micelares, lo que reduce su potencial higroscópico, que puede expresarse como el producto de la superficie interna por la afinidad [1], [13].

El contenido de humedad equilibrio ( $\mathrm{CHE}$ ), depende de la temperatura del aire circundante, de la humedad relativa y de las condiciones de secado a las cuales haya sido sometida previamente la madera. Maderas que son aparentemente iguales, generalmente no alcanzan el mismo contenido de humedad de equilibrio bajo idénticas 
condiciones atmosféricas, debido a las variaciones en los contenidos de extractivos o condiciones previas de secado [3], [16]. En Ucayali (Perú), entre una densidad básica de 0,70 a $0,20 \mathrm{~g} / \mathrm{cm}^{3}$ el contenido de humedad equilibrio varía de 12,5 a $15,5 \%$ para maderas como ana caspi (Apuleia leiocarpa (Vogel) J.F. Macbr.), aucatadijo (Croton matourensis Aubl.), sapote (Matisia cordata Bonpl.), utucuro (Septotheca tessmannii Ulbr.), pashaco blanco (Schizolobium parahyba (Vell:) S. F. Blake) y marupa (Simarouba amara Aubl.). Asimismo se tiene un contenido de humedad equilibrio para peine de mono (Apeiba membranacea Aubl.) 14,1 \%, manchinga (Brosimun utile (Kunth) Oken) $13 \%$ y achihua (Jacaranda copaia (Aubl.) D. Don) 13,7 \% [14].

Los niveles de humedad están en función de la densidad básica de la madera, porque cuando la humedad se incrementa desde $0 \%$ pasando por el contenido de humedad equilibrio hasta el punto de saturación de las fibras, aumenta moderadamente la masa anhidra y el volumen verde, pero la densidad básica disminuye levemente. Si continúa subiendo la humedad por encima del punto de saturación de las fibras, contenido de humedad libre hasta el contenido de humedad máximo, el volumen verde y densidad básica se hace constante, pero la masa anhidra sigue creciendo [12], [18], [20]. Por ello, conocer y entender los diversos niveles de contenido de humedad de la madera es importante en los procesos de secado e impregnación para obtener una materia prima de calidad en la elaboración de productos [8]. Los modelos matemáticos, constituyen instrumentos de alta utilidad para la industria forestal particularmente del secado y preservación de la madera, porque genera cálculos rápidos y prácticos [8] , [16] , [23].

Por lo indicado anteriormente el objetivo principal propuesto es determinar los modelos matemáticos que interpreta el comportamiento de las maderas, relacionando el punto de saturación de las fibras, contenido de humedad libre, contenido de humedad máxima y contenido de humedad equilibrio en función de la densidad básica. Problema fundamental que por su escasa información de estos niveles de humedad es necesario su investigación. Con la hipótesis alterna plateada siguiente: los niveles de humedad de las maderas relacionadas con la densidad básica poseen diferente contenido de humedad equilibrio a $12 \%$, punto de saturación de las fibras a $30 \%$, contenido de humedad libre $30 \%$ y contenido de humedad máxima que $60 \%$.

\section{Materiales y métodos}

La investigación se realizó en la xiloteca de la Universidad Nacional Amazónica de Madre de Dios de la ciudad de Puerto Maldonado, Madre de Dios (Perú), ubicada a $12^{\circ} 36^{\prime} \mathrm{S}$ y $77^{\circ} 32^{\prime} \mathrm{O}$. Una temperatura media anual de $26,7{ }^{\circ} \mathrm{C}$ y HR media anual de $84,7 \%$ [19]. El tipo de investigación fue orientada y método de investigación correlacional. De las 150 especies diferentes con estudios físicos de la madera que se tuvo como población, aplicando la fórmula de Arkin y Colton [6], la muestra estimada fue 105 especies del cual $10 \%$ fue para ensayos y $90 \%$ para validación, desarrollándose el estudio en cuatro etapas.

\section{Primera etapa}

Consistió en recopilar y buscar información sobre propiedades físicas de la madera en forma oficial y publicada (tesis e informes especializados) de 105 maderas tropicales de universidades e instituciones de investigación peruana.

\section{Segunda etapa}

Consistió en hacer los ensayos físicos de diez especies (cuadro 1): Apeiba tibourbou Aubl. D. DOM, Brosimum lactescens (S. Moore) C.C. Beg., Cedrelinga cateniformis (Ducke) Ducke, Eschweilera coriácea (DC.) S.A. Mori, Guazuma ulmifolia Lam., Jacaranda copaia (Aubl.) D. DOM, Manilkara bidentata (A. DC.) A. Chev., Spondias mombin L, Swietenia macrophylla King y Tabebuia serratifolia (Vahl) G. Nicholson, seleccionados al azar, considerando dos especies por cada uno de los cinco grupos de densidad básica clasificada por [2]. Se saturó probetas de $25 \times 25 \times 100$ $\mathrm{mm}$ en agua caliente a $100{ }^{\circ} \mathrm{C}$ por un periodo de 24 horas, luego se sometió al secado en horno $103^{\circ} \mathrm{C} \pm$ $2{ }^{\circ} \mathrm{C}$, hasta alcanzar peso constante [11], [12]. La masa saturada y anhidra se tomó mediante una balanza digital (precisión 0,01 g) y su volumen saturado y anhidro por inmersión en agua [4]. Los valores se calcularon con las ecuaciones 1 y 2 .

$$
\mathrm{DB}=\mathrm{Mo} / \mathrm{Vs}
$$

Donde: DB es la Densidad básica de la madera ( $\mathrm{g} /$ $\mathrm{cm}^{3}$ ). Mo es Masa anhidra de la probeta (g). y Vs es Volumen de la probeta saturada $\left(\mathrm{cm}^{3}\right)$.

$$
C V=\{(V s-V o) / V s\} * 100
$$

Donde: Cv es la Contracción volumétrica de la probeta en estado verde. Vo es Volumen de la probeta al estado anhidro. Vs es Volumen de la probeta saturada en $\mathrm{cm}^{3}$. 
El cálculo del contenido de humedad máximo (CHM) se realizó con la metodología desarrollada por [24], consistió en someter las mini probetas $(25 \times 25 \times 5$ $\mathrm{mm}$ ) a saturación en agua a $100^{\circ} \mathrm{C}$ por un periodo de 4 horas, se determinó su masa mediante una balanza digital (precisión 0,01 g) y su volumen por inmersión en agua [4]. Posteriormente se secaron en un horno a $103 \pm 2{ }^{\circ} \mathrm{C}$, durante 48 horas para obtener su masa anhidra, método conocido como desplazamiento de agua (fluidos) desarrollado por [20]. Se determinó con la ecuación 3.

$$
\mathrm{CHM}=\{(\mathrm{Ms}-\mathrm{Mo}) / \mathrm{Mo}\}{ }^{*} 100
$$

CHM es Contenido de humedad máximo (\%). Ms esMasa saturada en agua (g). Mo es la Masa anhidra (g).

Considerando la contracción volumétrica y la densidad básica desarrollada por [22], se determinó el punto de saturación de las fibras (PSF) empleando el factor de ajuste de la densidad del agua en la pared celular $(1,113)$ propuesta por [9] con la ecuación 4.

$$
\mathrm{PSF}=1,113 \mathrm{CV} / \mathrm{DB}
$$

PSF es Punto de saturación de las fibras. CV es Contracción volumétrica (\%). DB es Densidad básica $\left(\mathrm{g} / \mathrm{cm}^{3}\right)$.

La determinación del contenido de humedad libre $(\mathrm{CHL})$ se realizó con lo propuesto por [23], que se obtiene con la ecuación 5 .

$$
\mathrm{CHL}=\mathrm{CHM}-\mathrm{PSF}
$$

$\mathrm{CHL}$ es Contenido de humedad libre de la madera (\%)

Para determinar el contenido de humedad equilibrio (CHE) se usó probetas de 25×100×150 mm. Se empleó el método de medición instantánea [12], que consistió en introducir el higrómetro de pines en la cara de las probetas en las 10 espacies maderables una vez por semana, repitiéndose cada semana durante el periodo de un año.

\section{Tercera etapa}

Consistió en determinar los modelos matemáticos de ajuste para interpretar el comportamiento de las maderas relacionando la densidad básica con los niveles de humedad (CHL, CHM, PSF y CHE). Utilizando para ello la regresión lineal, logarítmica, exponencial y potencial con auto correlación de Durbin y Watson, recomendados por [9], [12], [23].

\section{Cuarta etapa}

Consistió en validar la investigación, utilizando para ello los datos tecnológicos de propiedades físicas de las 95 especies forestales, cuya variable utilizada fue la densidad básica para determinar los niveles de humedad con los modelos matemáticos generados en la investigación.

El procesamiento y análisis de datos, se realizó con el paquete estadístico SPSS - 19, última versión en español en conjunto con Microsoft Excel. El análisis de relación se determinó conociendo el coeficiente de determinación ( $r 2$ ) y coeficiente de correlación (r) más elevado. Ante este último [6], indica que hay una correlación significativa entre dos variables cuantitativas, si el coeficiente de correlación $r \geq 0,7$ o si $r \leq-0,7$. Así mismo se realizó el análisis de varianza de Fisher por modelo matemático seleccionado con el fin de determinar su significancia al $\mathrm{P}<0,05 \circ 95 \%$ de certeza.

\section{Resultados y discusión}

El cuadro 1 muestra el nivel de humedad calculado y ajustado en función de la densidad básica de la madera de 10 maderas ensayadas. Donde los valores del contenido de humedad libre (CHL) corroboran en algunos géneros y especies dentro del rango encontrado por [24] al señalar que varía de 14 a $238 \%$ para un rango de densidad básica de 0,29 a $1,05 \mathrm{~g} / \mathrm{cm}^{3}$. Estos valores de $\mathrm{CHL}$ explicarían que en los procesos de impregnación representaría el máximo de solución preservante que es capaz de absorber una madera y que en los procesos de secado representa el agua que se puede eliminar por capilaridad sin el requerimiento de elevada energía [23]. El agua libre al encontrarse en los lúmenes de las células, ésta queda fuertemente influida por el volumen de los espacios vacíos, es decir a mayor proporción del volumen mayor es la cantidad de agua libre que una determinada especie pueda contener, es por ello que puede llegar a representar un peso de hasta cuatro veces más que la madera y como explica también [23].

Los valores del contenido de humedad máxima (CHM) coincide con lo señalado por [15], [21], quienes indican que Krugiodendron ferrum con una densidad de 1,18 alcanza un $\mathrm{CHM}$ de $26 \%$ y, en contraste, Ochroma lagopus con una densidad de 0,2 puede llegar a presentar $400 \%$ o más. Igualmente coincide con [23], 
Cuadro 1. Nivel de humedad calculado y ajustado en función de la densidad básica de la madera.

Table 1. Humidity level calculated and adjusted according to the basic wood density.

\begin{tabular}{|c|c|c|c|c|c|c|c|c|c|}
\hline \multirow{3}{*}{ Especies } & \multirow{3}{*}{$\frac{\mathrm{DB}}{\mathrm{g} / \mathrm{cm}^{3}}$} & \multicolumn{8}{|c|}{ Nivel de humedad (\%) } \\
\hline & & \multicolumn{4}{|c|}{ Calculado } & \multicolumn{4}{|c|}{ Ajustado } \\
\hline & & $\mathrm{CHE}$ & PSF & $\mathrm{CHL}$ & $\mathrm{CHM}$ & $\mathrm{CHE}$ & PSF & $\mathrm{CHL}$ & $\mathrm{CHM}$ \\
\hline J. copaia (achihua) & 0,266 & 15,02 & 68,27 & 241,86 & 310,13 & 15,38 & 44,04 & 245,10 & 280,70 \\
\hline A. tibourbou (peine de mono) & 0,287 & 15,79 & 36,60 & 240,76 & 277,36 & 15,06 & 41,37 & 229,00 & 264,56 \\
\hline S. mombin (ubos) & 0,384 & 14,99 & 27,54 & 169,29 & 196,83 & 13,85 & 32,46 & 166,66 & 200,58 \\
\hline G. ulmifolia (bolaina negra) & 0,399 & 13,70 & 27,52 & 146,39 & 173,91 & 13,70 & 31,48 & 159,01 & 192,54 \\
\hline S. macrophylla (caoba) & 0,488 & 11,10 & 20,87 & 117,59 & 138,45 & 12,87 & 26,65 & 119,04 & 149,61 \\
\hline C. cateniformis (tornillo) & 0,659 & 11,05 & 20,39 & 57,10 & 77,49 & 11,62 & 20,74 & 67,97 & 91,81 \\
\hline B. lactescens (manchinga) & 0,685 & 11,57 & 20,35 & 56,40 & 76,75 & 11,46 & 20,10 & 62,53 & 85,37 \\
\hline T. serratifolia (tahuari) & 0,740 & 9,47 & 18,30 & 43,36 & 61,66 & 11,14 & 18,84 & 52,17 & 72,91 \\
\hline E. coriacea (misa blanca) & 0,779 & 12,38 & 20,21 & 36,16 & 56,37 & 10,93 & 18,06 & 45,99 & 65,33 \\
\hline M. bidentata (quinilla) & 0,919 & 11,18 & 18,27 & 15,90 & 34,17 & 10,24 & 15,75 & 29,16 & 43,92 \\
\hline
\end{tabular}

que dicen que son menores de $65 \%$ las maderas nativas de densidades mayores a $0,75 \mathrm{~g} / \mathrm{cm}^{3}$ y mayor a $250 \%$ maderas con densidades menores de $0,30 \mathrm{~g} / \mathrm{cm}^{3}$.

El modelo del punto de saturación de las fibras (PSF) difiere a lo encontrado por [23], pero indica que hay similitud que para valores de $0,29 \mathrm{~g} / \mathrm{cm}^{3}$ le corresponde $35 \%$ y para $1,05 \mathrm{~g} / \mathrm{cm} 3$ le corresponde un $16 \%$. Sin embargo la relación del PSF en caoba, ubos, manchinga y tahuari fue menor en dos unidades e indica que varía del 13 al $39 \%$ para 1,05 a $0,29 \mathrm{~g} / \mathrm{cm}^{3}$ de densidad básica. Tal situación puede explicarse al hecho de que el PSF sobre todo en especies latifoliadas tropicales presenta un rango de variación amplio toda vez que de acuerdo con [10], [15], señalan que tal cambio es debido a la presencia, cantidad, tamaño y fundamentalmente extractivos tienen un PSF relativamente bajo. Asimismo [13], señala que maderas extremadamente ligeras pueden llegar a tener un PSF de hasta $50 \%$. Por su parte [10], reportan valores de hasta $15 \%$, que coincide con la investigación. También [17], indica que el agua higroscópica comienza a salir de la madera a altos contenidos de humedad a 51 $\%$ en tornillo, $36 \%$ en pumaquiro y $77 \%$ en huairuro y no a partir de $30 \%$. [1], señala que la saturación de las fibras comienza a $40 \%$ para el haya, $41 \%$ para el abedul amarillo, $43 \%$ para congona y $56 \%$ para cachimbo en presencia de agua líquida. Finalmente [7], manifiesta que la pérdida total del agua libre en arce, se daba recién a un contenido de humedad de $14 \%$.

Los valores del contenido de humedad equilibrio (CHE) corrobora a lo encontrado en Chile por [16], que indica que en un rango de 0,62 a $0,30 \mathrm{~g} / \mathrm{cm}^{3}$ le corresponde de 11,7 a $17,8 \%$. De igual forma para maderas peruanas [14], señala que para 0,70 a $0,20 \mathrm{~g} / \mathrm{cm}^{3}$ le corresponde de 12,5 a 15,5 \% encontrándose aquí el ana caspi, sapote, utucuro, pashaco blanco y marupa, así como el peine de mono con $14,1 \%$, manchinga $13 \%$ y achihua $13,7 \%$.

La figura 1 muestra la relacion de los valores calculados y ajustados de los niveles de humedad en función de la densidad básica de la madera, donde el contenido de humedad equilibrio $(r=0,8505 ; P<0,05)$ y el punto de saturación de las fibras $(r=0,8787 ; P<0,05)$ tienen un coeficiente de correlación alta, mientras que el contenido de humedad máxima $\quad(r=0,9896 ; P<0,05)$ $y$ el contenido de humedad libre $(r=0,9882 ; P<0,05)$ presenta un coeficiente de correlación muy alta. Estos resultados son coherentes porque se encuentran dentro de los valores hallados por [7] , [9] , [14] y [17] para algunas especies forestales tropicales peruanas y mexicanas. Asimismo, se tiene un comportamiento exponencial para el contenido de humedad máxima igual a $\mathrm{CHM}=597,96 \mathrm{e}^{-2,843 \mathrm{BB} \text { y }}$ el contenido de humedad libre igual a $\mathrm{CHL}=583,84 \mathrm{e}^{-3,263 \mathrm{DB}}$. Mientras que un comportamiento potencial igual a PSF $=14,678 \mathrm{DB}-0,83$ y un comportamiento logarítmico para el contenido de humedad equilibrio igual a $\mathrm{CHE}=-4,141 \mathrm{ln}(\mathrm{DB})+9,8939$.

El cuadro 2 muestra los resultados de la aplicación de los modelos matemáticos determinados con las 10 maderas ensayadas, con el fin de validar y realizar la predicción de los niveles de humedad en función de la densidad básica de la madera de las 95 especies forestales, valores que se ajustan mejor y se aproximan a los encontrados por [9], [14], [17] y [27].

La figura 2 muestra que la relación de los valores de validación de las 95 especies forestales de los niveles de humedad (CHE, PSF, CHL y CHM) ajustados presenta mejor asociación que los niveles de humedad calculados 

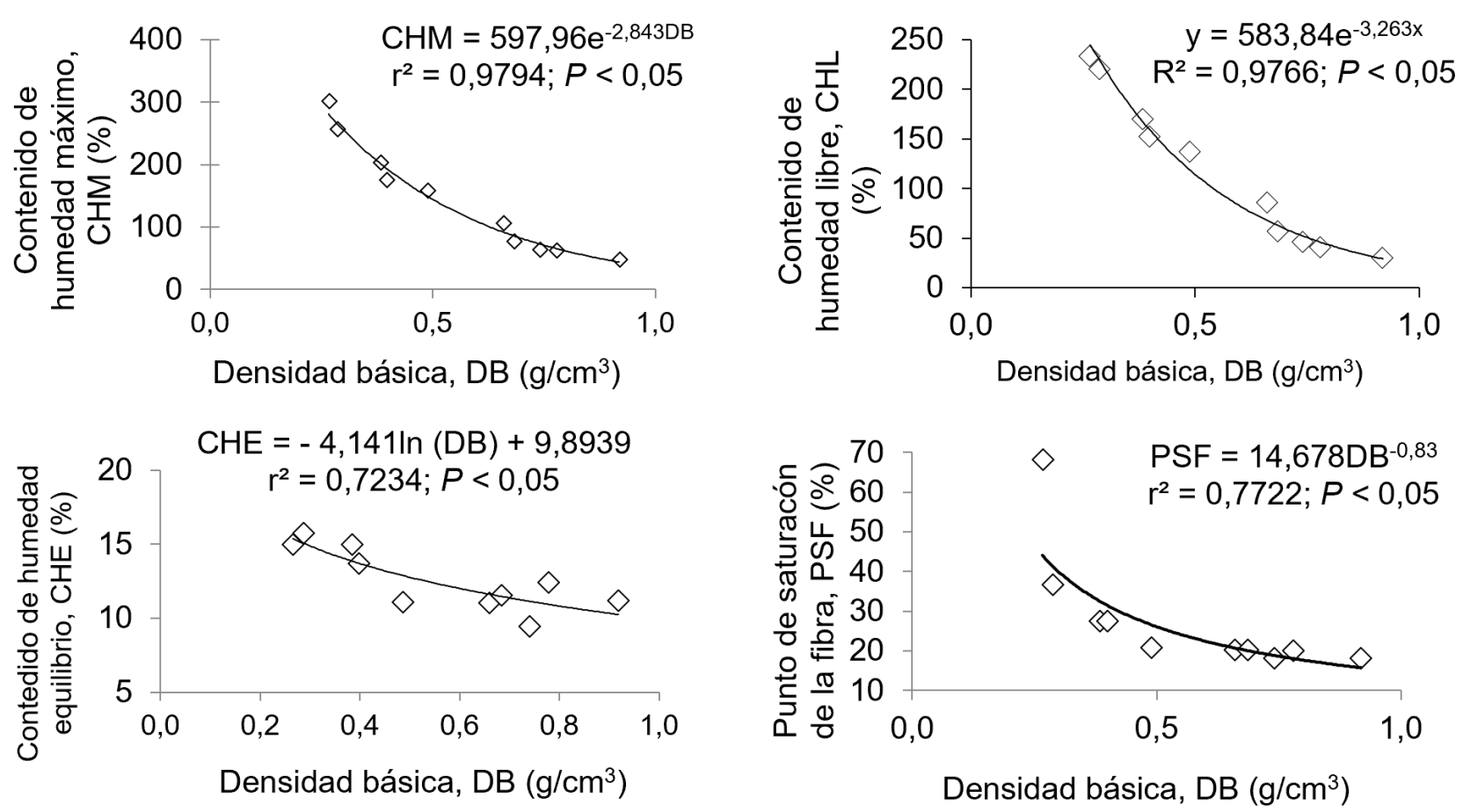

Figura 1. Relación de la densidad básica (DB) con los niveles de humedad (CHL, CHM, PSF y CHE) de 10 especies de Madre de Dios.

Figure 1. Relation of the basic density (DB) with the humidity levels (CHL, CHM, PSF and CHE) of 10 species of Madre de Dios.
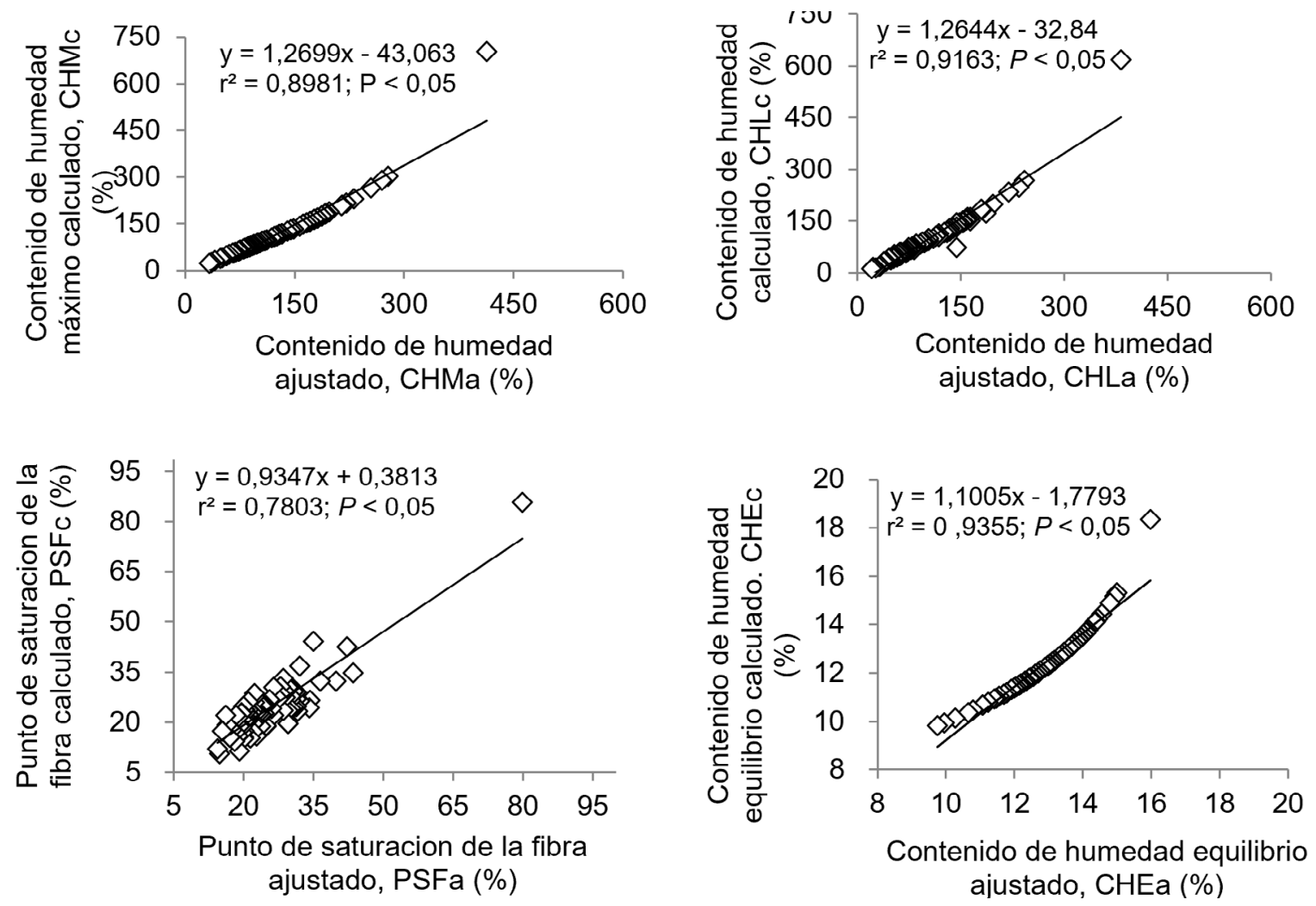

Figura 2. Relación de 95 maderas con los niveles de humedad (CHE, PSF, CHM y CHL) calculados (c) versus los niveles de humedad ajustados (a) por los modelos.

Figure 2. Ratio of 95 woods with the humidity levels (CHE, PSF, CHM and CHL) calculated (c) versus moisture levels adjusted (a) by the models. 
Cuadro 2. Predicción de niveles de humedad en función de la densidad básica de la madera.

Table 2. Prediction of humidity levels based on the basic wood density.

\begin{tabular}{|c|c|c|c|c|c|c|}
\hline \multirow{2}{*}{ No } & \multirow{2}{*}{ Especie } & \multirow{2}{*}{$\frac{\mathrm{DB}}{\mathrm{g} / \mathrm{cm} 3}$} & \multicolumn{4}{|c|}{ Niveles de humedad (\%) } \\
\hline & & & $\mathrm{CHE}$ & PSF & $\mathrm{CHM}$ & $\mathrm{CHL}$ \\
\hline 1 & Ochroma pyramidale (Cav. Ex Lam) Urban (topa) & 0,13 & 18,34 & 79,82 & 413,26 & 382,06 \\
\hline 2 & Apeiba membranacea Spruce (maquisapa ñaccha 1) & 0,27 & 15,32 & 43,51 & 277,60 & 242,00 \\
\hline 3 & Chorisia integrifolia Ulbr. (lupuna blanca) & 0,28 & 15,17 & 42,22 & 269,82 & 234,23 \\
\hline 4 & Vochysia sp. (quillosisa) & 0,30 & 14,88 & 39,87 & 254,92 & 219,44 \\
\hline 5 & Coussapoa sp. (ubilla) & 0,33 & 14,43 & 36,47 & 231,44 & 196,40 \\
\hline 6 & Virola albdiflora (cumala 1) & 0,35 & 14,24 & 35,08 & 221,15 & 186,42 \\
\hline 7 & Simarouba amara Aubl. (marupa) & 0,36 & 14,12 & 34,27 & 214,95 & 180,44 \\
\hline 8 & Endlicheria williamsii O. C. (isma moena) & 0,36 & 14,11 & 34,19 & 214,34 & 179,85 \\
\hline 9 & Bombax paraense Ducke (punga) & 0,39 & 13,79 & 32,07 & 197,38 & 163,62 \\
\hline 10 & Sclerolobium sp. (ucshaquiro blanco) & 0,39 & 13,79 & 32,07 & 197,38 & 163,62 \\
\hline 11 & Malmea sp. (espintana) & 0,40 & 13,72 & 31,60 & 193,50 & 159,92 \\
\hline 12 & Croton lechleri Muell. Arg. (sagre de grado) & 0,40 & 13,69 & 31,40 & 191,85 & 158,36 \\
\hline 13 & Micranta spruceana (Baillon) R. Schultes (higuerilla) & 0,40 & 13,69 & 31,40 & 191,85 & 158,36 \\
\hline 14 & Sapium marmieri Huber (caucho masha) & 0,40 & 13,69 & 31,40 & 191,85 & 158,36 \\
\hline 15 & Schizolobium excelsum Vogel (pashaco 2) & 0,40 & 13,69 & 31,40 & 191,85 & 158,36 \\
\hline 16 & Guazuma crinita C. Mart. (bolaina blanca) & 0,41 & 13,59 & 30,76 & 186,48 & 153,28 \\
\hline 17 & Hura crepitans L. (catahua amarilla) & 0,41 & 13,59 & 30,76 & 186,48 & 153,28 \\
\hline 18 & Pithecellobium pedicellare (Dc.) Bentham (vilco colorado) & 0,41 & 13,59 & 30,76 & 186,48 & 153,28 \\
\hline 19 & Podocarpus sp. (romerillo macho) & 0,41 & 13,59 & 30,76 & 186,48 & 153,28 \\
\hline 20 & Cedrela montana Moritz ex Turczaninov (cedro virgen) & 0,42 & 13,49 & 30,16 & 181,25 & 148,36 \\
\hline 21 & Cedrela odorata L. (cedro) & 0,42 & 13,49 & 30,16 & 181,25 & 148,36 \\
\hline 22 & Nectandra sp. (moena negra) & 0,42 & 13,49 & 30,16 & 181,25 & 148,36 \\
\hline 23 & Croton rimbachii Croizat.(sangre de grado 1) & 0,43 & 13,40 & 29,63 & 176,68 & 144,07 \\
\hline 24 & Amburana cearensis (Ducke) A. C. Smith. (ishpingo) & 0,43 & 13,39 & 29,57 & 176,17 & 143,60 \\
\hline 25 & Ficus sp. (oje renaco) & 0,43 & 13,39 & 29,57 & 176,17 & 143,60 \\
\hline 26 & Matisia cordata Humb. Et Bonpl. (zapote) & 0,43 & 13,39 & 29,57 & 176,17 & 143,60 \\
\hline 27 & Poulsenia armata (Mlguel) Standley (yanchama) & 0,44 & 13,29 & 29,01 & 171,24 & 138,99 \\
\hline 28 & Thopis sp. (shachavaca micuna) & 0,44 & 13,29 & 29,01 & 171,24 & 138,99 \\
\hline 29 & Albizzia sp. (pashaco) & 0,45 & 13,20 & 28,48 & 166,44 & 134,53 \\
\hline 30 & Virola sebifera Aubl. (cumala blanca) & 0,45 & 13,20 & 28,48 & 166,44 & 134,53 \\
\hline 31 & Guatteria hyposericeae (carahuasca) & 0,46 & 13,11 & 27,96 & 161,78 & 130,22 \\
\hline 32 & Otaba parvifolia (aguanillo) & 0,46 & 13,11 & 27,96 & 161,78 & 130,22 \\
\hline 33 & Tethorchidium rubrivenium (col de monte) & 0,46 & 13,11 & 27,96 & 161,78 & 130,22 \\
\hline 34 & Zanthoxylum sp. (hualaja) & 0,47 & 13,02 & 27,47 & 157,24 & 126,04 \\
\hline 35 & Brosimun utile (H.B.K.) Pitt. (panguana) & 0,49 & 12,85 & 26,53 & 148,56 & 118,08 \\
\hline 36 & Gallesia integrifolia (Spreng) Harms. (palo ajo) & 0,49 & 12,85 & 26,53 & 148,56 & 118,08 \\
\hline 37 & Ficus glabrata H.B.K. (oje rosado) & 0,50 & 12,76 & 26,09 & 144,39 & 114,29 \\
\hline 38 & Calophyllum brasiliense Camb. (lagarto caspi) & 0,51 & 12,68 & 25,67 & 140,35 & 110,62 \\
\hline 39 & Hevea brasiliensis (H.B.K) Huelt (shiringa) & 0,53 & 12,52 & 24,86 & 132,59 & 103,63 \\
\hline 40 & Ocotea costulata (casho moena) & 0,53 & 12,52 & 24,86 & 132,59 & 103,63 \\
\hline 41 & Poducarpus oleifolius Don in Lamb. (diablo fuerte) & 0,53 & 12,52 & 24,86 & 132,59 & 103,63 \\
\hline 42 & Carapa guienesis Aubl. (andiroba) & 0,54 & 12,45 & 24,48 & 128,88 & 100,31 \\
\hline
\end{tabular}

Continúa en la siguiente página ... 


\begin{tabular}{|c|c|c|c|c|c|c|}
\hline \multirow{2}{*}{ No } & \multirow{2}{*}{ Especie } & \multirow{2}{*}{$\begin{array}{c}\mathrm{DB} \\
\mathrm{g} / \mathrm{cm} 3\end{array}$} & \multicolumn{4}{|c|}{ Niveles de humedad (\%) } \\
\hline & & & $\mathrm{CHE}$ & PSF & $\mathrm{CHM}$ & $\mathrm{CHL}$ \\
\hline 43 & Parahancornia amapa (Huber) Ducke (naranjo podrido) & 0,54 & 12,45 & 24,48 & 128,88 & 100,31 \\
\hline 44 & Matisia bicolor Ducke (machin sapote) & 0,55 & 12,38 & 24,18 & 125,98 & 97,72 \\
\hline 45 & Ocotea sp. (roble corriente) & 0,55 & 12,37 & 24,11 & 125,27 & 97,09 \\
\hline 46 & Iryanthera laevis Margrak (cumala colorada) & 0,56 & 12,30 & 23,79 & 122,10 & 94,28 \\
\hline 47 & Aniba amazonica Meiz (moena amarilla) & 0,56 & 12,29 & 23,75 & 121,76 & 93,97 \\
\hline 48 & Seiba samauma (Mart.) Schum. (huimba) & 0,57 & 12,22 & 23,40 & 118,34 & 90,96 \\
\hline 49 & Cariniana domesticata Mart. (cachimbo) & 0,59 & 12,08 & 22,74 & 111,81 & 85,21 \\
\hline 50 & Clarisia racemosa R. y P. (mashonaste) & 0,59 & 12,08 & 22,74 & 111,81 & 85,21 \\
\hline 51 & Aspidosperma vargasii A. DC. (quillabordon) & 0,60 & 12,01 & 22,43 & 108,67 & 82,48 \\
\hline 52 & Guarea kurthiana Adr. Juss. (requia) & 0,60 & 12,01 & 22,43 & 108,67 & 82,48 \\
\hline 53 & Pouteria sp. (caimito) & 0,60 & 12,01 & 22,43 & 108,67 & 82,48 \\
\hline 54 & Rheedia sp. (charichuelo) & 0,60 & 12,01 & 22,43 & 108,67 & 82,48 \\
\hline 55 & Copaifera officinalis L. (copaiba) & 0,61 & 11,94 & 22,12 & 105,63 & 79,83 \\
\hline 56 & Ormosia coccinea Jacks. (huayruro) & 0,61 & 11,94 & 22,12 & 105,63 & 79,83 \\
\hline 57 & Protium sp. (copal) & 0,61 & 11,93 & 22,09 & 105,33 & 79,57 \\
\hline 58 & Pterygota sp. (paujil ruro) & 0,62 & 11,87 & 21,83 & 102,67 & 77,27 \\
\hline 59 & Pseudolmedia laevis (Ruiz \& Pav.) (chimicua sin pelo) & 0,63 & 11,81 & 21,54 & 99,79 & 74,79 \\
\hline 60 & Terminalia sp. (roble amarillo) & 0,64 & 11,77 & 21,40 & 98,38 & 73,58 \\
\hline 61 & Virola sp. (pucuna caspi) & 0,64 & 11,77 & 21,37 & 98,10 & 73,34 \\
\hline 62 & Caryocar coccineum Pilg. (almendro) & 0,65 & 11,68 & 20,99 & 94,28 & 70,07 \\
\hline 63 & Sickingia tinctorea (HBK) K. Schum. (huacamayo caspi) & 0,65 & 11,68 & 20,99 & 94,28 & 70,07 \\
\hline 64 & Tetragastris altíssima (Aubl.) Swartz (isigo) & 0,65 & 11,68 & 20,99 & 94,28 & 70,07 \\
\hline 65 & Couratari macrosperma A. C. Schmidt (papelillo caspi) & 0,65 & 11,67 & 20,93 & 93,74 & 69,61 \\
\hline 66 & Sloanea sp. (cepanchina) & 0,65 & 11,67 & 20,93 & 93,74 & 69,61 \\
\hline 67 & Brosimum aubletti P.y E. (Tamamuri) & 0,66 & 11,61 & 20,72 & 91,64 & 67,82 \\
\hline 68 & Aspidosperma macrocarpon Mart. (pumaquiro) & 0,67 & 11,55 & 20,47 & 89,07 & 65,64 \\
\hline 69 & Inga sp. (shimbillo) & 0,67 & 11,55 & 20,47 & 89,07 & 65,64 \\
\hline 70 & Manilkara sp. (balata) & 0,67 & 11,53 & 20,36 & 88,06 & 64,79 \\
\hline 71 & Brosimun alicastrum Swartz (congona) & 0,68 & 11,49 & 20,22 & 86,57 & 63,53 \\
\hline 72 & Mezilaurus itauba (Meissner) Taubert ex Mez. (itahuba) & 0,68 & 11,49 & 20,22 & 86,57 & 63,53 \\
\hline 73 & Ptecarpus sp. (palo sangre amarillo) & 0,68 & 11,49 & 20,22 & 86,57 & 63,53 \\
\hline 74 & Peltogyne sp. (palo violeta) & 0,69 & 11,43 & 19,97 & 84,15 & 61,50 \\
\hline 75 & Pseudolmedia laevigata Trécul (chimicua con pelo) & 0,69 & 11,43 & 19,97 & 84,15 & 61,50 \\
\hline 76 & Trichilia sp. (uchumullaca) & 0,69 & 11,43 & 19,97 & 84,15 & 61,50 \\
\hline 77 & Apuleia molaris Spruce ex Bentham (ana caspi) & 0,70 & 11,37 & 19,73 & 81,79 & 59,52 \\
\hline 78 & Tetragastris panamensis O. Kuntze (copal) & 0,70 & 11,37 & 19,73 & 81,79 & 59,52 \\
\hline 79 & Heisteria sp. (yutubanco) & 0,71 & 11,31 & 19,50 & 79,50 & 57,61 \\
\hline 80 & Pseudomedia laevis (R y P) Macbr. (chimicua) & 0,71 & 11,31 & 19,50 & 79,50 & 57,61 \\
\hline 81 & Ptecarpus sp. (palo sangre negro) & 0,73 & 11,20 & 19,06 & 75,10 & 53,97 \\
\hline 82 & Terminalia amazonia (J. F. Gmelin) Exell.(yacushapana 1) & 0,73 & 11,20 & 19,06 & 75,10 & 53,97 \\
\hline 83 & Diplotropis martiusii Bent in Mart. (chontaquiro) & 0,74 & 11,14 & 18,85 & 73,00 & 52,24 \\
\hline 84 & Lucuma dolichophylla Standley. (quina quina) & 0,74 & 11,14 & 18,85 & 73,00 & 52,24 \\
\hline 85 & Calycophyllum spruceanum Benth. (capirona) & 0,76 & 11,03 & 18,43 & 68,97 & 48,94 \\
\hline 86 & Hymenaea sp. (azucar huayo 1) & 0,77 & 10,97 & 18,19 & 66,65 & 47,06 \\
\hline
\end{tabular}




\begin{tabular}{|c|c|c|c|c|c|c|}
\hline \multirow{2}{*}{ No } & \multirow{2}{*}{ Especie } & \multirow{2}{*}{$\frac{\mathrm{DB}}{\mathrm{g} / \mathrm{cm} 3}$} & \multicolumn{4}{|c|}{ Niveles de humedad (\%) } \\
\hline & & & $\mathrm{CHE}$ & PSF & $\mathrm{CHM}$ & $\mathrm{CHL}$ \\
\hline 87 & Myroxilon balsamun Harms. (estoraque) & 0,78 & 10,92 & 18,04 & 65,16 & 45,85 \\
\hline 88 & Tabebuia capitata (Bur y Sch) Sandw (tahuari 1) & 0,81 & 10,79 & 17,56 & 60,52 & 42,12 \\
\hline 89 & Crepidospermum goudotianum (palo baston) & 0,83 & 10,67 & 17,13 & 56,53 & 38,95 \\
\hline 90 & Aspidosperma sp. (quillabordon 1) & 0,83 & 10,67 & 17,13 & 56,53 & 38,95 \\
\hline 91 & Coumarouna odorata Aubl. (shihuahuaco) & 0,87 & 10,47 & 16,48 & 50,45 & 34,19 \\
\hline 92 & Sterculia sp. (manchari caspi) & 0,89 & 10,37 & 16,15 & 47,53 & 31,92 \\
\hline 93 & Myrcianthes fragans (Sw) McVaugh (capirona colorada) & 0,95 & 10,13 & 15,38 & 40,77 & 26,77 \\
\hline 94 & Brosimun paraense (palo sangre 1) & 0,99 & 9,94 & 14,80 & 35,87 & 23,11 \\
\hline
\end{tabular}

por formulas del ensayo, por ello el CHE ( $r=0,9672$; $\mathrm{P}<0,05), \mathrm{CHL}(r=0,9572 ; \mathrm{P}<0,05)$ y $\mathrm{CHM}(r=0,9476$; $\mathrm{P}<0,05)$ tienen un grado de asociación muy alta, mientras que el PSF $(r=0,8833 ; P<0,05)$ tiene una asociación alta, el mismo que se asemeja a lo encontrado por [9], [23].

\section{Conclusiones}

Es conveniente realizar más ensayos con nuevas especies maderables potenciales y en otras ciudades del país para así ajustar más los modelos, por densidad básica o peso específico de la pared celular.

\section{Agradecimentos}

A la Xiloteca de la Universidad Nacional Amazónica de Madre de Dios (Perú) por permitir utilizar sus muestras de madera y a las personas que de manera directa e indirecta apoyaron en el cumplimiento de objetivos de la presente investigación.

\section{Referencias}

[1] G. Almeida, "Influence of the structure of madera in addition propiedades físico-mecánicas con alto contenido de humedad", PhD thesis, University of Laval, Canadá, 2006.

[2] A. Aróstegui, Recopilación y análisis de estudios tecnológicos de maderas peruanas, Lima, Perú: Ministerio de Agricultura, Instituto Nacional Forestal y de Fauna, pp. 57, 1982.

[3] J. Arroyo. "Propiedades físico-mecánicas de la madera". Texto para estudiantes de Ingeniería Forestal. Facultad de ciencias forestales. Universidad de los Andes, Mérida,
Venezuela, pp. 197, 1983.

[4] ASTM (American Society of Testing Materials), D - 2395, "Standard test methods for specific gravity of wood and wood-based materials", ASTM International, West Conshohocken, USA, pp. 14, 1998.

[5] E. Bluhm, R. Rosende, W. G. Kauman, "Determinación de la humedad de equilibrio de la madera en todas las zonas climáticas de Chile". Informe Técnico 21. Instituto Forestal, Santiago, Chile, pp.136-143, 1965.

[6] Z. M. Córdova, Estadística descriptiva e inferencial, Perú: Pontificia Universidad Católica del Perú, pp. 450, 1997.

[7] M. De la Cruz, "Punto de saturación de las fibras es igual al $30 \%$ ", Comentarios sobre el punto de saturación de las fibras, Xilema, vol. 19, pp.30-32, 2007.

[8] J. J. Erdoiza, M. I. Castillo, "Susceptibilidad de impregnación con preservadores de cincuenta especies maderables mexicanas". Xalapa: Boletín técnico la madera y su uso. No. 22. 1989.

[9] S. M. Fuentes, "Estimación del punto de saturación de la fibra de las maderas", Revista Chapingo Serie Ciencia Forestales y del Ambiente, vol. 6, no. 1, pp. 79-81, 2000.

[10] J. G. Haygreen, L. J. Bowyer, "Forest products and wood science an introduction". lowa: Editorial Ames lowa State University Press, 3ra Edición, pp. 484, 1996.

[11] INDECOPI. Norma NTP 251.011. 2004, "Método para determinar la densidad”, Lima, Perú, pp. 9, 2004.

[12] JUNAC (Junta del acuerdo de Cartagena), "Manual del grupo andino para el secado de maderas", Colombia: Editorial Carbajal S. A, pp. 52, 1989.

[13] F. Kollmann, "Tecnología de la madera y sus aplicaciones", Madrid: Instituto Forestal de Investigaciones y Experiencias (IFIE), 1959.

[14] M. Lluncor, "Estudio de las propiedades físicas de diez especies maderables potenciales de bosques secundarios y primarios residuales", AIDER, Pucallpa, Perú, Informe técnico PD 512/08, rev. 2, no. 1, pp. 1-46, 2013.

[15] A. J. Panshin, C. Zeeuw, "Textbook of wood technology", New York, USA: McGraw-Hill, 1980. 
[16] P. N. Pérez, L, Valenzuela, V, Díaz, R, Ananías, "Predicción del contenido de equilibrio de la madera en función del peso específico de la pared celular y variables ambientales", Maderas, Ciencia y tecnología, vol. 13, no. 3, pp. 253-266, 2011.

[17] M. Pontín, "Propiedades físico mecánicas de tres espacies madereras tropicales por encima y por debajo del punto de saturación de las fibras", Tesis, Universidad Laval, Quebec, Canadá, 2005.

[18] E. Rosales, "Variabilidad del contenido de humedad equilibrio de la madera de diez especies comerciales para tres regiones del Perú", Revista Forestal Mesoamericana Kuru, vol. 13, no. 30, pp 13-21, 2016.

[19] SENAMHI (Servicio nacional de meteorología e hidrografía), "Datos climáticos de los últimos 30 años para Lima, Huánuco y Puerto Maldonado", Sede central, Lima, pp. 1-6, 2013.

[20] J. Siau. Transport processes in Wood. New York. Springer Series in Wood Science (ed. T.E. Timell). 1984.

[21] W. Simpson, A. Tenwolde. "Physical properties and moisture relations of Wood, Wood Handbook: Wood as an engineering material". USDA Forest Service, Forest Products Laboratory. Washington, USA, 1999.

[22] C. Skar, "Water in Wood", Syracuse University Press, p. 218, 1972.

[23] J. Tamarit, M. Fuentes, "Parámetros de humedad de 63 maderas mexicanas en función de su densidad básica", Revista Chapingo Serie Ciencias Forestales y del Ambiente, vol. 9, no. 2, pp. 156-164, 2003.

[24] L. Valenzuela, M. Ramírez, C. Levancine, A. Arevalo, "Investigación exploratoria, variación radial de la densidad de la pared celular en la madera de Pinus radiata D. Don de 29 años de edad, Valdivia Chile". Presentado en el II congreso de la Universidad Austral de Chile, 2004 
